\title{
Controlled Trial on the Effect of 10 Days Low-Frequency Repetitive Transcranial Magnetic Stimulation (rTMS) on Motor Signs in Parkinson's Disease
}

\author{
Pablo Arias, PhD, ${ }^{1}$ Jamile Vivas, $\mathrm{PhD},{ }^{1}$ Kenneth L. Grieve, $\mathrm{PhD},{ }^{1,2}$ and Javier Cudeiro, \\ $\mathrm{MD}, \mathrm{PhD}^{1}$ \\ ${ }^{1}$ Neuroscience and Motor Control Group (NEUROcom), Department of Medicine-INEF and Institute for Biomedical \\ Research (INIBIC), University of A Coruña, Spain \\ ${ }^{2}$ Faculty of Life Sciences (Neurosciences), The University of Manchester, Manchester, United Kingdom
}

\begin{abstract}
We evaluated the effect of low-frequency rTMS on motor signs in Parkinson's disease (PD), under a double-blind placebo-controlled trial design. PD patients were randomly assigned to received either real (n 59 ) or sham (n 5 9) rTMS for 10 days. Each session comprises two trains of 50 stimuli each delivered at $1 \mathrm{~Hz}$ and at $90 \%$ of daily rest motor threshold using a large circular coil over the vertex. The effect of the stimulation, delivered during the ON-period, was evaluated during both ON and OFF periods. Tests were carried out before and after the stimulation period, and again 1 week after. The effect of the stimulation was evaluated through several gait variables (cadence, step amplitude, velocity, the CVstride-time, and the turn time), hand dexterity, and also the total and motor sections of the UPDRS. Only the total and motor section of the UPDRS and the turn time during gait were affected by the stimulation, the effect appearing during either ON or OFF evaluation, and most importantly, equally displayed in both real and sham group. The rest of the variables were not influenced. We conclude the protocol of stimulation used, different from most protocols that apply larger amount of stimuli, but very similar to some previously reported to have excellent results, has no therapeutic value and should be abandoned. This contrasts with the positive reported effects using higher frequency and focal coils. Our work also reinforces the need for sham stimulation when evaluating the therapeutic effect of rTMS.
\end{abstract}

Key words: TMS; Parkinson's disease; therapy; motor recovery

The therapeutic role of repetitive transcranial magnetic stimulation (rTMS) in motor symptoms in Parkinson's disease (PD) is currently not well defined. Although there have been a significant number of studies performed, the heterogeneity in their protocols and conflicting results do not reveal clear conclusions about the effectiveness of the technique. However, studies involving single session rTMS ${ }^{1,2}$ suggest that repeated rTMS might offer significant improvements in parkinsonian patients. These studies have utilized a number of different paradigms, changing variables such as stimulus intensity, duration, frequency, location and even coil type, not forgetting the actual tests carried out, the drug status of the patients and the degree of disease severity. With multiple sessions the number of variables increases still further, greatly enlarging the parameter space. It is therefore unsurprising that studies utilizing repeated sessions of rTMS have shown mixed results, but including some apparent successes. ${ }^{3-8}$ An important aspect of many studies (largely overlooked) is the absence of a properly controlled placebo group. We therefore adopted an experimental paradigm, which attempts to build upon previously successful multiple session rTMS protocols, ${ }^{3,4,7}$ with the addition of an equally large randomly assigned control group, given "sham"' rTMS. Our study tested the effect of 10 sessions of low-frequency rTMS, stimulating patients with a large round coil over the vertex at a frequency of $1 \mathrm{~Hz}$ (utilized apparently successfully by Mally and Stone ${ }^{3,4}$ and Mally and colleagues ${ }^{7}$ ), but normalizing intensity as a function of RMT, in a doubleblind fashion. In short, we chose our protocol to try to replicate earlier work by Mally and colleagues, 3,4,7 while trying to change those methodological elements, which we felt accounted for their "positive", results. Also, given the suggestion of a ceiling effect of medication, 9 we evaluated motor symptoms during both ON and OFF phases, as done before with other stimulation protocols and target areas, ${ }^{8,10-12}$ (of 
important therapeutic relevance). Finally, since rTMS has been reported to be more effective when applied during the ON phase, ${ }^{8}$ we stimulated only during $\mathrm{ON}$.

\section{PATIENTS AND METHODS}

\section{Patients}

From a pool of 25 volunteer patients with PD, 19 met the criteria (see later) and 18 (nine real, nine sham) completed the study (Fig. 1). Patients had to match the primary diagnosis of PD (based on medical records following established diagnostic criteria); being at stages II-IV according to the criteria of Hoehn and Yahr during OFF; and be able to cope with OFF periods. Patients were not taking antidepressant medication. Exclusion criteria were as follows: previous experience with TMS, implanted devices, pumpbased drug administration systems, history of seizures, dementia (MMSE < 24), arthromuscular deficit or joint prostheses, presence of dyskinesias disturbing stimulation or motor evaluation, unpredictable motor fluctuations, and medication change within the month prior to the start of the experiment.

Patients were required to complete all sessions and could not change their medication regime during the study. They enrolled into the program in groups of up to four patients every 3 weeks and were randomly assigned to receive real or sham stimulation. The distribution of the two last groups were counterbalanced (based on UPDRS score), avoiding a possible imbalance due to chance.

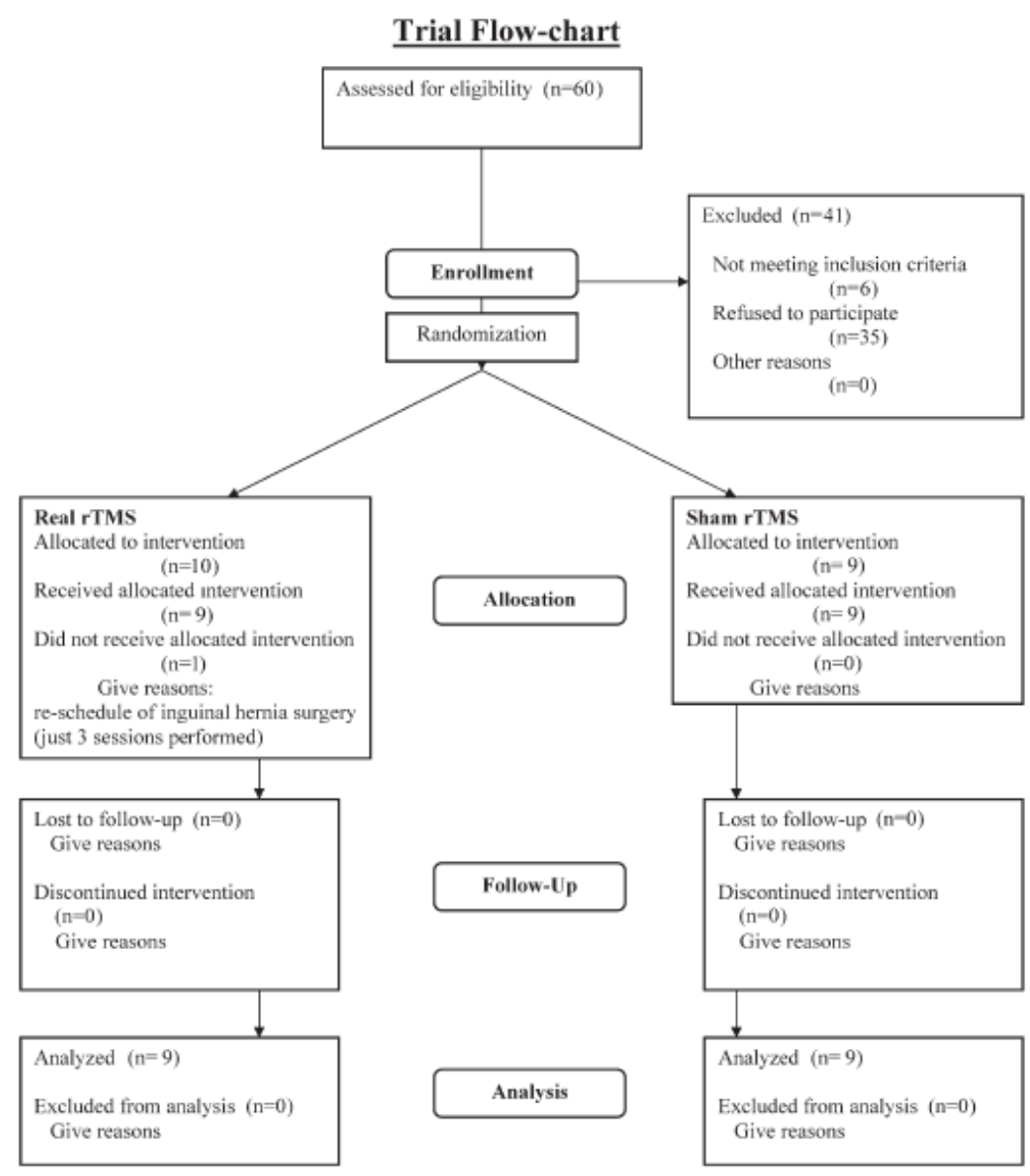

FIG. 1. Trial flow-chart. 


\section{Procedure}

Motor evaluations were carried out three times: before the 10 stimulation sessions (PRE), after stimulation sessions (POST), and 1 week after POST (POST-2).

Evaluations were performed at weekends, 1 day in OFF, the other in ON; allocated to ON-OFF or OFF-ON after randomization. This order was reproduced at POST and POST-2.

ON evaluation was performed when the effect of medication was optimal, based on patients selfreport $^{13}$ and neurological examination.

OFF evaluation was performed after one night with holding medication ( $\geq 12$ hours) (10 patients took controlled release drugs at the last intake of the day, which was not taken the day before OFF evaluation; five patients wore transdermal patches which were removed the day before OFF evaluation, $\geq 12$ hours); OFF was confirmed by patients and neurological evaluation.

TMS (Monday to Friday for 2 weeks) was applied in the morning during self-rated ON periods, between 1 and 2 hours after medication intake. ${ }^{8}$ The protocol was in accord with the Helsinki declaration and approved by the University of A Coruña ethics committee. Patients signed consent forms.

\section{Evaluations}

Motor tests—walking, hand dexterity, and motor section of the UPDRS.

- Walking: Walk 5 m, turn around (left), walk back 5 m, turn around (right), and walk 5 m. Each trial therefore comprised $15 \mathrm{~m}$ and two turns. Subject performed this three times, resting 2 minutes between trials. The task was performed using the preferred walking pattern. The instruction given was: "Walk along the corridor as you normally do, turn around the cone at the end, come back walking as you normally do, turn around this other cone, and go back again walking as you normally do to touch the button on the wall', (Fig. 2). Before the three recording trials, one practice trial was performed.

- Hand dexterity utilized the Purdue Pegboard. Subjects had to place as many pegs as possible, sequentially into a row of holes within 30 seconds. The number of pegs was recorded. Subjects performed this three times for each hand.

- UPDRS: The total score and the motor section of the scale were taken into account.

- Evaluation was undertaken by authors PA and JV, blind to the stimulation protocol.

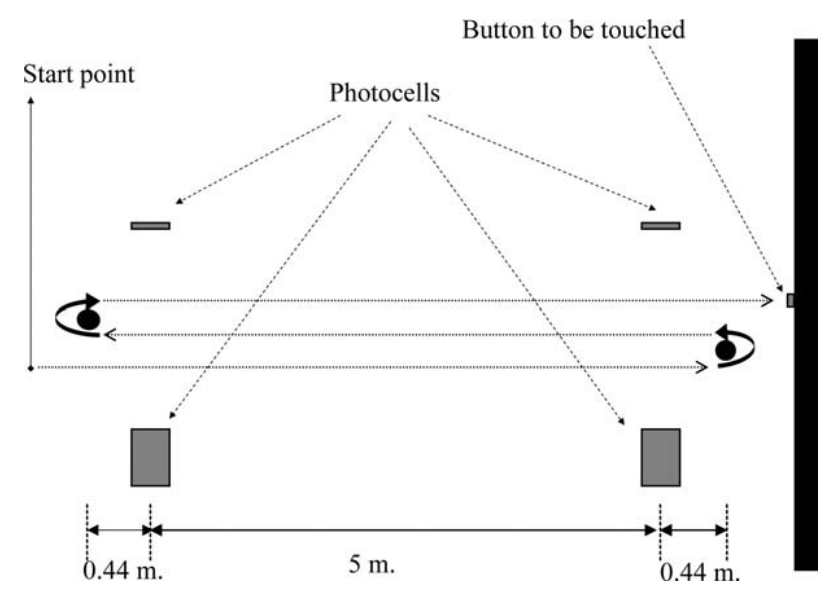

FIG. 2. Representation of the gait procedure.

\section{Stimulation}

Stimulation was applied from behind, with a round coil (see apparatus) centered over the vertex (by authors K.L.G. and J.C.). Each session, 100 pulses were delivered in trains of 50 at $1 \mathrm{~Hz}$. One was delivered clockwise and the other anticlockwise with a 5-minute pause inbetween. Intensity was set at $90 \%$ of rest motor threshold (RMT) defined as the lowest intensity eliciting a clear response of $50 \mu \mathrm{V}$ peak-topeak amplitude in the first dorsal interosseus of the less affected side, in 5/10 of successive trials. This was measured daily before stimulation. 
For sham stimulation, two coils were used. One coil, unconnected to the stimulator, was placed over the vertex (as earlier). The second coil (connected to the stimulator, but set at 35\% output) was placed in direct contact to the first, but tilted $90^{\circ}$. Thus, the patient could feel only one coil flat over the head (as had been explained to all patients prior to the study). During the sham protocol, EMG settings were maintained and some single pulses were delivered (mimicking RMT determination). We have now been made aware of the availability of specific placebo sham coils. ${ }^{14}$

\section{Apparatus}

For gait evaluation, the recording system comprised footswitches worn as insoles in the shoes connected to a radio-transmitter attached to the subjects' belt. Data (sampled at $1 \mathrm{KHz}$ ) were sent to a computer receiver allowing stride cycle time to be registered. ${ }^{15}$ Two photocells, placed 5-m apart, were connected to the recording system (Fig. 2).

Hand dexterity was measured as described earlier. TMS stimulation used a High Power $90 \mathrm{~mm}$ round coil powered by a Magstim Rapid (The Magstim Company, Whiyland, UK). Motor-evoked potentials (MEP) were registered using Ag/Cl surface electrodes attached with a belly tendon montage.

\section{Analyzed Variables}

For gait:

- Velocity, time to cover the straight parts of the task, data obtained from the photocells.

- Cadence, calculated from data obtained through footswitches, corresponding to the straight part of the task.

- Step amplitude, derived from velocity and cadence.

- Turn time, obtained from the successive activation of the photocells (before and after turns).

- The CVstride-time, obtained from the footswitches data of the straight section, defined as follows:

$$
\mathrm{CV}(\%)=(\mathrm{SD} / \mathrm{mean}) \times 100
$$

All gait values were the mean of three trials. Values for manual dexterity were the averages for the three trials of the Peg Board test, averaged across both hands. Total and motor scores from the UPDRS were analyzed.

\section{Data Analysis}

To check homogeneity between groups in PRE a Students ' $t$ '” for independent samples was applied for each variable; either in ON or OFF.

To evaluate stimulation effectiveness, a three-way ANOVA with repeated measures was performed for each variable; two within-subjects factors were defined, evaluation with three levels (PRE, POST, and POST-2), and drug with two levels (ON and OFF). The between subjects factor was group (real or sham stimulation). For total score of UPDRS (assessed during ON), suppressing factor drug reduced to a twoway ANOVA with repeated measures.

Normality of distribution was check by K-S test. Greenhouse-Geisser correction was applied when sphericity was violated. SPSS was used for statistical analysis. Significance was set at $P<0.05$.

\section{RESULTS}

\section{Groups Homogeneity: PRE Real Versus Sham}

In the PRE tests, both groups, real and sham, were not significantly different for all analyzed variables (in ON and OFF periods, see Fig. 3 and Table 1). Thus, at the beginning of the program, real and sham groups had comparable grades of "disability." 

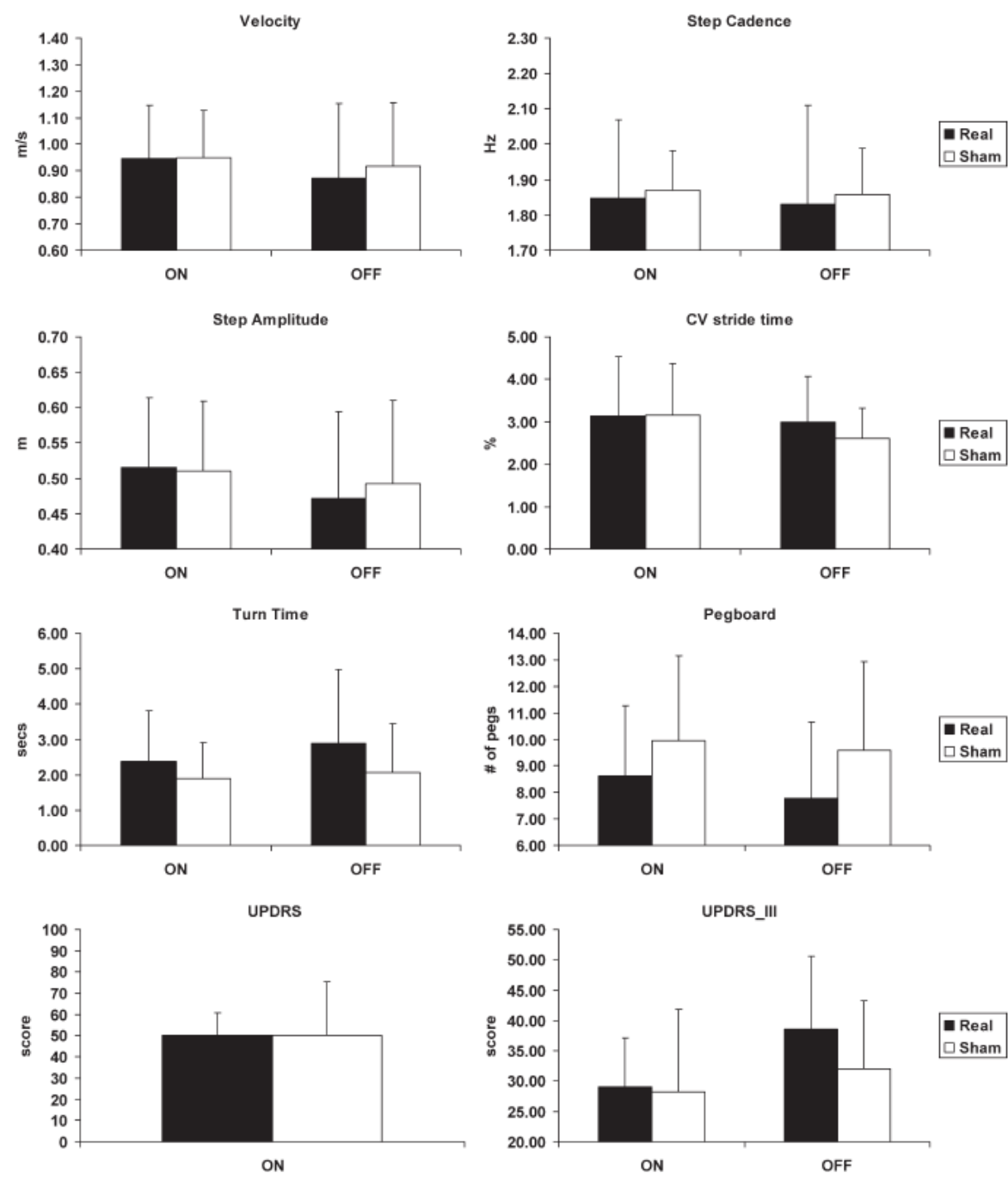

FIG. 3. Values (means, SD) for each variable before stimulation (PRE). Black bars, real stimulation; open bars, sham stimulation. There were no significant differences between groups at PRE for any variable, either during ON or OFF periods (total score of UPDRS was assessed only during $\mathrm{ON}$ ). Groups were therefore comparable before stimulation (see also Table 1).

\section{Effect of the Stimulation Protocol}

\section{Variables Not Affected by the Protocol}

A number of variables were unaffected by rTMS, in either ON or OFF tests, and this was seen in both real and the sham groups: step amplitude, cadence, velocity, and the $\mathrm{CV}_{\text {stride-time. }}$ The same was seen for hand dexterity (Fig. 4 and Table 2). Evaluation * drug interaction was not significant in any variable, proving the lack of effect was not dependent on the status of the medication, but also proving TMS did not alter drug influence on the PD (Table 2). No significant interaction involving group was detected, so the aforementioned description occurred for both groups, real and sham (Table 2).

There were some significant effects of factor drug, showing the effectiveness of medication: step amplitude $F(1,16)=4.749, P=0.045$, and for the Pegboard $F(1,16)=6.837, P=0.019$. In other tests, the effect of drug did not reach significance; in both cases, these effects were observed in both groups.

These results show that the rTMS protocol did not affect these variables. 
TABLE 1. Groups homogeneity

\begin{tabular}{lcc}
\hline & $\mathrm{t}$ & $P$-value \\
\hline Gait velocity & $t(16)=0.027$ & \\
ON & $t(16)=0.378$ & $P=0.979$ \\
OFF & $t(16)=0.249$ & $P=0.710$ \\
Step cadence & $P=0.807$ \\
ON & $t(11.241)=0.277$ & $P=0.787$ \\
OFF & $t(16)=0.098$ & \\
Step amplitude & $t(16)=0.356$ & $P=0.923$ \\
ON & $t(16)=0.011$ & $P=0.726$ \\
OFF & $t(13.752)=0.920$ & $P=0.991$ \\
CV stride time & $t(16)=0.838$ & $P=0.374$ \\
ON & $t(16)=0.991$ & $P=0.414$ \\
OFF & & $P=0.336$ \\
Turn time & $t(16)=0.973$ & \\
ON & $t(16)=1.229$ & $P=0.345$ \\
OFF & $t(16)=0.036$ & $P=0.237$ \\
Pegboard & & $P=0.971$ \\
ON & $t(16)=0.147$ & $P=0.885$ \\
OFF & $t(16)=1.198$ & $P=0.248$ \\
UPDRS & & \\
ON & & \\
UPDRS-III & & \\
ON & & \\
OFF & & \\
\hline
\end{tabular}

PRE real versus sham. Between groups (real vs. sham) before stimulation (PRE). $t$ and associated $P$-values are shown in ON and OFF periods, except UPDRS, evaluated only during ON. Lack of significance shows groups were comparable at PRE, before the stimulation period.

\section{Variables Affected by the Protocol}

Conversely, some other variables were indeed affected by the protocol, as a main effect of factor evaluation was seen (Table 2). When this happened, the evaluation * drug interaction was always not significant (Table 2), proving the effect of the protocol was present to the same extent either in ON or OFF phases, thus answering one of our stated objectives.

However, the key element involved possible interactions with factor group. No significant interaction involving Group was seen (Table 2), proving behavior of sham and real groups were the same, suggesting a strong placebo effect as cause of improvement in the signs (Fig. 4, UPDRS_III and the TT during gait, see Table 2 for a summary). A significant effect of factor drug was also in the UPDRS_III $(F(1,16)=$ 25.846, $P<0.001$ ) proving a better performance in presence of medication; this was not achieved for TT.

Finally, for all variables analyzed, affected by the protocol or not, the factor group was not significant for any variable [logical, since groups were homogenous at the beginning (PRE) and all effects (significant or not) affected both groups similarly (see earlier and Table 2)].

\section{DISCUSSION}

The primary objective of this study was a re-examination of the results obtained by previous studies showing significant beneficial effects on patients with PD using low-frequency rTMS, ${ }^{3,4}$ but with the addition of a properly balanced placebo group of "untested" " patients, evaluated using a blind paradigm. A secondary objective was to examine the possibility that the medication and its timetabling with respect to TMS could cause a "ceiling" effect, such that the true "effect" of rTMS would not be seen. Therefore, we decided to evaluate the effect of the treatment both in ON and OFF periods. , $^{8,10-12}$

Dealing with the second objective first, the results we obtained showed that effects did not depend on medication status. Our results match reasonably well those of Tergau et al. ${ }^{9}$ who could not prove effects of stimulation on motor function with the same type of coil, using high and low frequencies. Our data supports also earlier findings by Lomarev et al. ${ }^{8}$ (who actually used a different coil and stimulation 
frequency). It is also worth repeating that our stimulation was performed when patients were in the ON phase, following the report by Lomarev et al. ${ }^{8}$ that this was more effective rather than in the OFF phase.
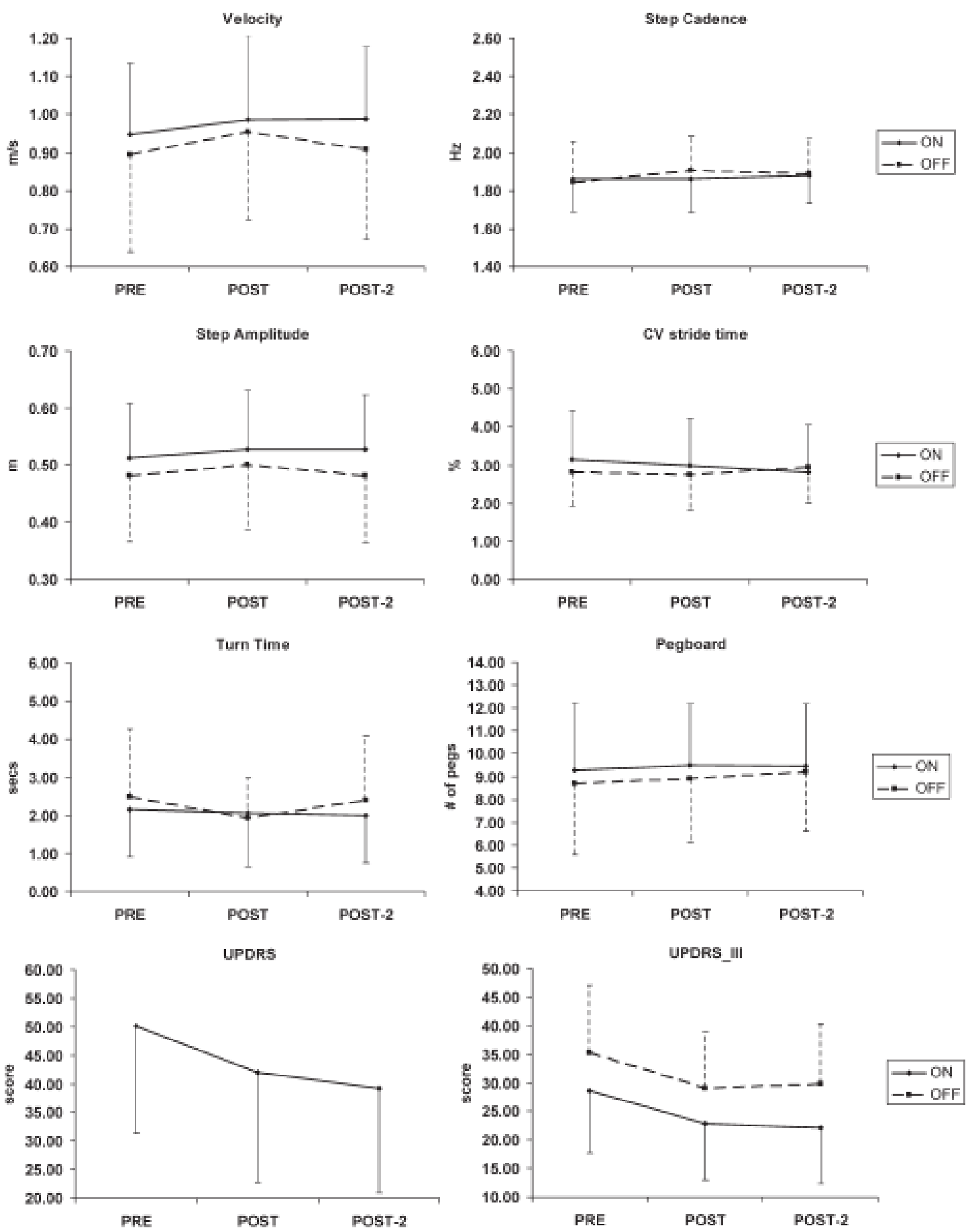

FIG. 4. The effect of the rTMS on the different variables before starting the period of stimulation (PRE), after the 10 sessions of stimulation (POST), and 1 week after finishing the period of stimulation (POST-2). Solid ON, dashed OFF (total score of UPDRS assessed during ON). Mean and SD are plotted for both groups pooled as no significant interactions involving factor group (realsham) were found (see also Table 2). 
TABLE 2. Effect of the stimulation protocol

\begin{tabular}{|c|c|c|c|c|c|}
\hline & Evaluation & Drug & Group & Evaluation * drug & $\begin{array}{c}\text { Evaluation * drug * group } \\
\text { (for UPDRS evaluation * group) }\end{array}$ \\
\hline Gait velocity & $\begin{array}{c}F(1.193,19.082)=3.472, \\
P=0.072\end{array}$ & $F(1,16)=2.694, P=0.120$ & $F(1,16)=0.000, P=0.996$ & $F(2,32)=2.129, P=0.136$ & $F(2,32)=0.943, P=0.400$ \\
\hline Step cadence & $F(2,32)=2.172, P=0.131$ & $F(1,16)=0.431, P=0.521$ & $F(1,16)=0.000, P=0.987$ & $F(2,32)=1.719, P=0.195$ & $F(2,32)=0.078, P=0.925$ \\
\hline Step amplitude & $\begin{array}{c}F(1.175,18.794)=1.758 \\
P=0.202\end{array}$ & $F(1,16)=4.749, P=0.045$ & $F(1,16)=0.001, P=0.973$ & $\begin{array}{c}F(1.492,23.866)=1.123 \\
P=0.326\end{array}$ & $\begin{array}{c}F(1.492,23.866)=1.123 \\
P=0.326\end{array}$ \\
\hline CV stride time & $F(2,32)=0.228, P=0.797$ & $F(1,16)=0.467, P=0.504$ & $F(1,16)=0.009, P=0.925$ & $\begin{array}{c}F(1.499,23.992),=0.117 \\
P=0.833\end{array}$ & $\begin{array}{c}F(1.499,23.992)=0.117 \\
P=0.833\end{array}$ \\
\hline Turn time & $F(2,32)=3.334, P=0.048$ & $F(1,16)=1.335, P=0.265$ & $F(1,16)=0.232, P=0.637$ & $F(2,32)=1.436, P=0.252$ & $F(2,32)=0.283, P=0.756$ \\
\hline Pegboard & $F(2,32)=1.982, P=0.152$ & $F(1,16)=6.837, P=0.019$ & $F(1,16)=1.652, P=0.217$ & $F(2,32)=0.541, P=0.587$ & $F(2,32)=1.080, P=0.352$ \\
\hline UPDRS & $F(2,32) 513.077, P<0.001$ & $\begin{array}{l}\text { NA, it was assessed } \\
\text { during ON periods }\end{array}$ & $F(1,16)=0.737, P=0.403$ & $\begin{array}{l}\text { NA, it was assessed } \\
\text { during ON periods }\end{array}$ & ${ }^{\mathrm{a}} F(2,32)=0.704, P=0.502$ \\
\hline UPDRS-III & $F(2,32)=24.470, P<0.001$ & $F(1,16)=25.846, P<0.001)$ & $F(1,16)=0.355, P=0.560$ & $F(2,32)=0.226, P=0.799$ & $F(2,32)=1.482, P=0.242$ \\
\hline
\end{tabular}

The values in bold indicate significant effects.

*Interaction evaluation * group for UPDRS, assessed only during ON periods. Turn time, UPDRS total and motor section were significantly affected by stimulation independently of ON or OFF. Importantly, evaluation * drug * group was not significant, showing that the effect was equal in real and sham. 
Notably, our main objective was to reinvestigate the effectiveness or otherwise of a low-frequency rTMS stimulation protocol, which appeared to offer significant therapeutic success, coupled with minimal patient inconvenience, as previously published by Mally et al. ${ }^{3,4}$ If an effect of rTMS shows therapeutic power, it seems likely that it will also be dependent on dose quantity, ${ }^{16,17}$ so that given reported safety guidelines, ${ }^{18}$ a protocol with low-stimulation frequency would be advantageous. In our case, however, we added a controlled for possible placebo effects. Our data clearly shows that the low frequency, short stimulus train was no more effective than the placebo, therefore this protocol has no therapeutic effect on motor signs in PD, calling into question the results of the previous studies.

Many of the variables analyzed involving gait (cadence, step amplitude, velocity, and variability of stride cycle time), and hand dexterity were unaffected by stimulation; while for those affected, improvements seem due to a placebo response. This was clearly seen in turn time, UPDRS motor section and total score. This contrasts with other results using treatment with circular coils applied over the vertex, ${ }^{3,4,7}$ but agrees with others. ${ }^{9,19}$

Reasons explaining these discrepancies may be several. In some studies, Mally et al. used a different stimulation intensity to ours-indeed, they did not individualize intensity to motor thresholds ${ }^{.47}$ Moreover, elsewhere they used 20\% of RMT,3 significantly lower than our 90\%. Other possibilities lie in technical details of coil and TMS delivery. Kammer et al. ${ }^{20}$ showed effects related to monophasic versus biphasic pulses; different pulse waveforms affect neurophysiological variables, ${ }^{21,22}$ for instance differentially affecting determination of motor threshold. ${ }^{23}$ We delivered biphasic pulses, whereas Mally and Stone ${ }^{3}$ used a model allowing waveform selection, but did not report the waveform used. While the differences could be explained if they had used monophasic pulses, their waveform would then be the same as used by Okabe et al., ${ }^{19}$ who like us, saw no beneficial results from low-frequency stimulation. Another variable is current direction. ${ }^{24}$ We applied both clockwise and anticlockwise to have the same effect over both hemispheres, much like Tergau et al. ${ }^{9}$ and Okabe et al.19 Mally and coworkers ${ }^{3,4,7}$ did not report direction. The number of pulses per day in our protocol was 100, the protocol being similar to others cited earlier. ${ }^{3,4,7,19}$ Tergau et al. ${ }^{9}$ utilized more pulses (1000), but with negative results; and it is therefore important to point out while interhemispheric excitability changes were shown to be associated with rTMS frequency, this might be independent of pulse number. ${ }^{25}$ Lang et al. ${ }^{26}$ showed differential changes in some neurophysiological variables using two different figure of eight coils (Magstim or Dantec) suggesting a possible explanation for different results, as we used Magstim and Mally Dantec. However, our results with Magstim are similar to those using Dantec. ${ }^{9}$ Thus, we must emphasize that the most noticeable difference between the "successful" underdosed studies and ours remains our use of the double blind, placebo-controlled experimental paradigm, which Mally and coworker did not use.

Basically, our results contrast with some other controlled studies which reported improvement in parkinsonian signs, using figure-of-eight coils and high stimulation frequencies. ${ }^{5,6,8}$ Clearly, the type of coil can have a role ${ }^{27,28}$; Lomarev et al. ${ }^{8}$ and Khedr et al. ${ }^{5,6}$ used figure-of-eight (focal) coils to stimulate the motor cortex with significant results. While accepting that the studies by Khedr et al. ${ }^{5,6}$ and Lomarev et al. ${ }^{8}$ were well controlled, we understand the stimulation protocols used by them are difficult to control with sham protocols, given that they used an intensity of $100 \%$ RMT.

We agree that future experiments should be performed to explore the therapeutic role of rTMS in PD (e.g., see Fregni et al. ${ }^{29}$ ), but argue that such studies should focus on higher frequencies and/or longer durations of stimulation; also patterned stimulation ${ }^{30}$ and combination of rTMS and tDCS deserves to be considered. ${ }^{31,32}$ In all cases, however, the necessity of appropriate controls, as for example concluded by Okabe et al. ${ }^{19}$ has been reinforced by our study, for this purpose new placebo coils (with somatosensory stimulation ${ }^{14}$ ) seem to be optimal in case of crossover designs, or when subjects are not naive to rTMS, which is not the case in this study.

Acknowledgments: This work was supported by Xunta de Galicia (Consellería de Educación2007/000140-0 and Dirección Xeral de I+D+i; PGIDIT06PXIC137004PN), Spain. We thank Dr. J.J. Díaz Silva and the physiotherapists Ms. Ana Novo and Ms. Soraya Núñez for their contribution to this work.

Financial Disclosures: Founding sources: Xunta de Galicia (Consellería de Educación-2007/0001400; Dirección Xeral de I+D+i PGIDIT06PXIC137004PN and INCITE09 137379 PR) and MICIIN (BFU2009-08169), Spain. 
Author Roles: P. Arias: Conception, organization, and execution of research project, design, execution, and review and critique of statistical analysis, and writing of the first draft, and review and critique of the manuscript. J. Vivas: Execution of research project, review and critique of statistical analysis, and review and critique of the manuscript. K. L. Grieve: Execution of research project, review and critique of statistical analysis, and writing of the first draft and review and critique of the manuscript. J. Cudeiro: Conception, organization, and execution of research project, design, execution, and review and critique of statistical analysis, and writing of the first draft, and review and critique of the manuscript.

\section{REFERENCES}

1. Siebner HR, Mentschel C, Auer C, Conrad B. Repetitive transcranial magnetic stimulation has a beneficial effect on bradykinesia in Parkinson's disease. Neuroreport 1999;10:589-594.

2. Lefaucheur JP, Drouot X, Von Raison F, Ménard-Lefaucheur I, Cesaro P, Nguyen JP. Improvement of motor performance and modulation of cortical excitability by repetitive transcranial magnetic stimulation of the motor cortex in Parkinson's disease. Clin Neurophysiol 2004;115:2530-2541.

3. Mally J, Stone TW. Improvement in Parkinsonian symptoms after repetitive transcranial magnetic stimulation. J Neurol Sci 1999;162:179-184.

4. Mally J, Stone TW. Therapeutic and “Dose-dependent" effect of repetitive microelectroshock induced by transcranial magnetic stimulation in Parkinson’s disease. J Neurosci Res 1999;57:935-940.

5. Khedr EM, Farweez HM, Islam H. Therapeutic effect of repetitive transcranial magnetic stimulation on motor function in Parkinson's disease patients. Eur J Neurol 2003;10:567-567.

6. Khedr EM, Rothwell JC, Shawky OA, Ahmed MA, Hamdy A. Effect of daily repetitive transcranial magnetic stimulation on motor performance in Parkinson’s disease. Mov Disord 2006;21:2201-2205.

7. Mally J, Farkas R, Tóthfalusi L, Stone TW. Long-term follow-up study with repetitive transcranial magnetic stimulation (rTMS) in Parkinson’s disease. Brain Res Bull 2004;64:259-263.

8. Lomarev MP, Kanchana S, Bara-Jimenez W, Iyer M, Wassermann EM, Hallett M. Placebo-controlled study of rTMS for the treatment of Parkinson's disease. Mov Disord 2006;21:325-331.

9. Tergau F, Wassermann EM, Paulus W, Ziemann U. Lack of clinical improvement in patients with Parkinson's disease after low and high frequency repetitive transcranial magnetic stimulation. Electroencephalogr Clin Neurophysiol Suppl 1999;51:281-288.

10. Buhmann C, Gorsler A, Bäumer T, et al. Abnormal excitability of premotor-motor connections in de novo Parkinson's disease. Brain 2004;127:2732-2746.

11. Shimamoto H, Takasaki K, Shigemori M, Imaizumi T, Ayabe M, Shoji H. Therapeutic effect and mechanism of repetitive transcranial magnetic stimulation in Parkinson's disease. J Neurol 2001;248 (Suppl 3):48-52.

12. Mir P, Matsunaga K, Gilio F, Quinn NP, Siebner HR, Rothwell JC. Dopaminergic drugs restore facilitatory premotor-motor interactions in Parkinson disease. Neurology 2005;64:1906-1912.

13. Nieuwboer A, De Weerdt W, Dom R, Bogaerts K, Nuyens G. Development of an activity scale for individuals with advanced Parkinson disease: reliability and “on-off” variability. Phys Ther 2000;80:1087-1096.

14. Rossi S, Ferro M, Cincotta M, et al. A real electro-magnetic placebo (REMP) device for sham transcranial magnetic stimulation (TMS). Clin Neurophysiol 2007;118:709-716.

15. Arias P, Cudeiro J. Effects of rhythmic sensory stimulation (auditory, visual) on gait in Parkinson's disease patients. Exp Brain Res 2008;186:589-601.

16. Touge T, Gerschlager W, Brown P, Rothwell JC. Are the aftereffects of low-frequency rTMS on motor cortex excitability due to changes in the efficacy of cortical synapses?. Clin Neurophysiol 2001;112:2138-2145.

17. Quartarone A, Bagnato S, Rizzo V, et al. Distinct changes in cortical and spinal excitability following highfrequency repetitive TMS to the human motor cortex. Exp Brain Res 2005;161:114-124.

18. Rossi S, Hallett M, Rossini PM, Pascual-Leone A; Safety of TMS Consensus Group. Safety, ethical considerations, and application guidelines for the use of transcranial magnetic stimulation in clinical practice and research. Clin Neurophysiol 2009;120:2008-2039.

19. Okabe S, Ugawa Y, Kanazawa I. Effectiveness of rTMS on Parkinson's Disease Study Group. 0.2-Hz repetitive transcranial magnetic stimulation has no add-on effects as compared to a realistic sham stimulation in Parkinson's disease. Mov Disord 2003;18:382-388.

20. Kammer T, Beck S, Thielscher A, Laubis-Herrmann U, Topka H. Motor thresholds in humans: a transcranial magnetic stimulation study comparing different pulse waveforms, current directions and stimulator types. Clin Neurophysiol 2001;112:250-258.

21. Di Lazzaro V, Oliviero A, Mazzone P, et al. Comparison of descending volleys evoked by monophasic and biphasic magnetic stimulation of the motor cortex in conscious humans. Exp Brain Res 2001;141:121-127.

22. Di Lazzaro V, Oliviero A, Pilato F, et al. The physiological basis of transcranial motor cortex stimulation in conscious humans. Clin Neurophysiol 2004;115:255-266.

23. Sommer M, Alfaro A, Rummel M, et al. Half sine, monophasic and biphasic transcranial magnetic stimulation of the human motor cortex. Clin Neurophysiol 2006;117:838-844.

24. Di Lazzaro V, Oliviero A, Saturno E, et al. The effect on corticospinal volleys of reversing the direction of current induced in the motor cortex by transcranial magnetic stimulation. Exp Brain Res 2001;138:268-273.

25. Gorsler A, Baümer T, Weiller C, Münchau A, Liepert J. Interhemispheric effects of high and low frequency rTMS in healthy humans. Clin Neurophysiol 2003;114:1800-1807. 
26. Lang N, Harms J, Weyh T, et al. Stimulus intensity and coil characteristics influence the efficacy of rTMS to suppress cortical excitability. Clin Neurophysiol 2006;117:2292-2301.

27. Di Lazzaro V, Oliviero A, Profice P, et al. Comparison of descending volleys evoked by transcranial magnetic and electric stimulation in conscious humans. Electroencephalogr Clin Neurophysiol 1998;109:397-401.

28. Di Lazzaro V, Oliviero A, Pilato F, et al. Descending volleys evoked by transcranial magnetic stimulation of the brain in conscious humans: effects of coil shape. Clin Neurophysiol 2002;113:114-119.

29. Fregni F, Simon DK, Wu A, Pascual-Leone A. Non-invasive brain stimulation for Parkinson's disease: a systematic review and meta-analysis of the literature. J Neurol Neurosurg Psychiatry 2005;76:1614-1623.

30. Koch G, Brusa L, Carrillo F, et al. Cerebellar magnetic stimulation decreases levodopa-induced dyskinesias in Parkinson disease. Neurology 2009;73:113-119.

31. Lang N, Siebner HR, Ernst D, et al. Preconditioning with transcranial direct current stimulation sensitizes the motor cortex to rapid-rate transcranial magnetic stimulation and controls the direction of after-effects. Biol Psychiatry 2004;56:634-639.

32. Grüner U, Eggers C, Ameli M, Sarfeld AS, Fink GR, Nowak DA. $1 \mathrm{~Hz}$ rTMS preconditioned by tDCS over the primary motor cortex in Parkinson's disease: effects on Brady kinesia of arm and hand. J Neural Transm 2010;117:207-216. 